\title{
Il valore inclusivo dell'ambiente di apprendimento digitale
}

\author{
di Federica Ceriani and Fiammetta Bilancini
}

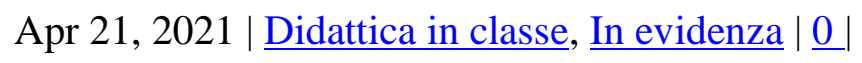

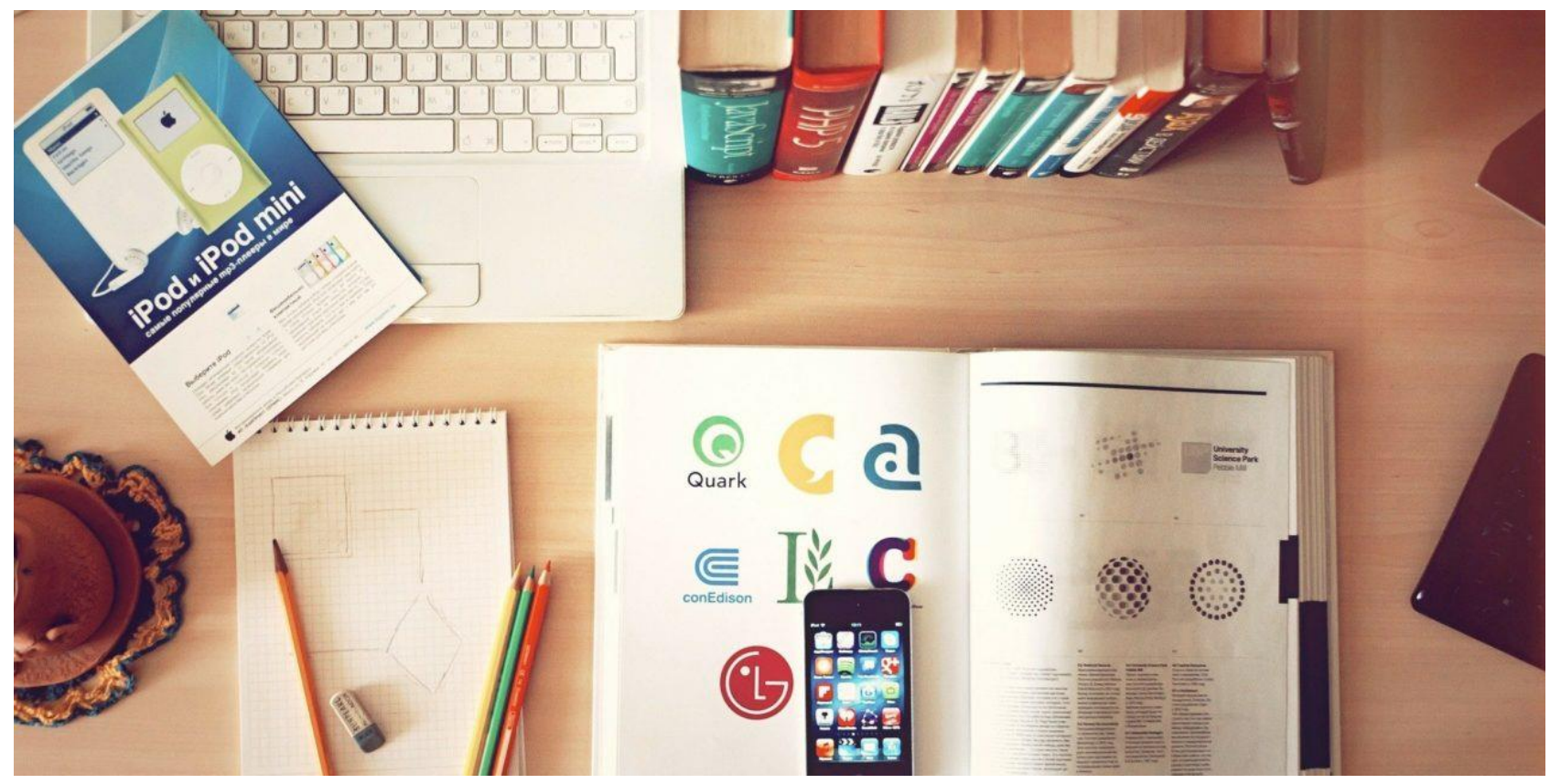

\begin{abstract}
Nell'articolo si ripropone il lavoro svolto nell' ambito del workshop Il valore inclusivo dell'ambiente di apprendimento, che può essere considerato il modello di base per la progettazione di nuovi percorsi in classe per l'insegnamento di Educazione Civica, in ambienti che non si limitino ad offrire esclusivamente strumenti compensativi e misure dispensative, ma che siano attenti a sviluppare i saperi, le abilità e le competenze di tutti gli studenti.
\end{abstract}

Inclusione dell'altro significa piuttosto che i confini della comunità sono aperti a tutti: anche - e soprattutto - a coloro che sono reciprocamente estranei e che estranei vogliono rimanere ${ }^{[1]}$.

Jürgen Habermas

Quando a scuola si usa il termine "inclusione" il pensiero corre veloce agli studenti disabili oppure a quelli stranieri o, in seconda battuta, agli studenti che provengono da situazioni socio-economiche svantaggiate. In questo breve articolo il termine "inclusione" si rifà ad un orizzonte di più ampio respiro. Come tutti i docenti ben sanno, ogni classe è una comunità di apprendimento, è il luogo 
dove la cittadinanza si fa attiva, è il contesto in cui ogni studente sperimenta la stretta connessione tra diritti e doveri, è l'ambiente dove si impara ad essere cittadini consapevoli e critici.

In questa cornice di senso, che cosa si intende per inclusione?

Inclusivo è lo sguardo capace di vedere nel compagno di classe che si ha di fronte un qualcuno che è diverso, altro da sé, e che al contempo fa parte di un mondo condiviso. Includere significa riconoscere ad ogni studente il diritto di argomentare le proprie opinioni, di manifestare una fitta rete di competenze in una forma personale e originale, di sviluppare una propria identità personale. Una classe, quindi, diventa inclusiva quando la comunità di apprendimento è aperta, quando è disposta non solo ad accogliere tutti i suoi membri, riconoscendone uguale valore e dignità, ma è propensa a valorizzare le peculiarità di ciascuno mettendole al servizio del bene comune.

\section{Per un ambiente di apprendimento inclusivo}

L'ambiente di apprendimento è un luogo della conoscenza ricco e complesso nel quale gli studenti trovano lo spazio per esprimere ed orchestrare le proprie conoscenze, abilità e competenze, dove la collaborazione è indirizzata al raggiungimento di un fine comune, dove la negoziazione di significati porta alla co-costruzione di nuovi saperi. Esso si compone di tre dimensioni tra loro correlate: la dimensione pedagogico-organizzativa, la dimensione metodologica e la dimensione relazionale.

\section{La dimensione pedagogico-organizzativa}

Questa dimensione riguarda l'organizzazione dello spazio fisico e dei tempi. Perchè l'ambiente di apprendimento diventi inclusivo, occorre prestare molta attenzione alla predisposizione dello spazio fisico: gli arredi devono essere disposti in modo da permettere la discussione e lo scambio di idee; i materiali devono essere alla portata di tutti gli studenti presenti nella classe; la cartellonistica e gli strumenti utili all'apprendimento possono essere costruiti dagli studenti stessi, in modo che diventino un primo approccio alla co-costruzione di saperi; è opportuno che i diversi spazi della scuola siano flessibili e siano a disposizione dell'operatività degli alunni. Grande importanza deve essere data anche alla scansione dei tempi: occorre prevedere che ci sia tempo per il dialogo e il confronto; è fondamentale rispettare i tempi di esecuzione di ciascuno studente; è necessario tutelare i tempi di realizzazione di un'attività dalla rigida scansione dell'orario scolastico. Appare, dunque, evidente come i docenti siano chiamati anzitutto a progettare, a disegnare lo spazio e il tempo: attraverso l'organizzazione di queste due categorie si compie il primo passo per rendere inclusivo l'ambiente di apprendimento.

\section{La dimensione metodologica}

Ogni docente ha nel proprio bagaglio esperienziale una serie di strumenti metodologici a disposizione. Sono i veri e propri "attrezzi da lavoro" senza i quali le proposte didattiche diventerebbero effimere attività, tese alla trasmissione di conoscenze e contenuti. L'utilizzo di metodologie attive e partecipative permette agli studenti di divenire i protagonisti effettivi dei molteplici percorsi di apprendimento proposti. Non solo, una scelta accurata di approcci metodologici cooperativi (cooperative learning, peer education, didattica della discussione, ecc.) permettono l'attivazione di vere pratiche inclusive: la collaborazione tra gli studenti e la messa in gioco delle differenti competenze individuali sono un elemento fondante delle didattiche innovative. L'inclusione, infatti, passa attraverso l'utilizzo consapevole e condiviso di una pluralità di strumenti di indagine, si avvale di dinamiche laboratoriali incentrate sul "fare insieme" (learning by doing), sviluppa la capacità di autovalutarsi e contribuire in modo concreto ed efficace al 
progetto condiviso.

I docenti, quindi, hanno il compito di scegliere in modo consapevole e ponderato le metodologie con le quali daranno vita e consistenza all'ambiente di apprendimento.

\section{La dimensione relazionale}

La terza dimensione, infine, sonda il delicato terreno del clima emotivo della classe. Agire sulle capacità relazionali degli studenti significa insegnare a decodificare e ad utilizzare gli strumenti comunicativi in modo tale da creare un clima di fiducia reciproca; significa costruire in modo partecipato un sistema di regole condiviso; significa educare all'ascolto, alla collaborazione e al dialogo costruttivo. Naturalmente l'inclusione è uno dei tratti caratterizzanti di questo quadro: la dimensione relazionale, infatti, porta con sé la presa di responsabilità di ogni componente della classe affinché ciascuno studente sia messo nella condizione di essere un protagonista attivo e partecipe del contesto di apprendimento. Ognuno, con le peculiarità che lo contraddistinguono, diviene un elemento indispensabile alla collettività.

I docenti, perciò, hanno il dovere di farsi carico del contesto relazionale della loro classe, mettendo in luce il valore aggiunto della collaborazione e l'importanza di percorsi di crescita condivisi.

In questo rapido excursus sulle tre dimensioni dell'ambiente di apprendimento appare evidente come il ruolo del docente sia un elemento chiave all'interno della classe. Accanto al ruolo di facilitatore delle dinamiche di apprendimento, infatti, egli è chiamato ad indossare i panni di designer dell'ambiente di apprendimento 2 .

\section{La Didattica a Distanza}

Con la sospensione delle attività didattiche in presenza, a seguito dell'emergenza Covid-19 e l'attivazione della didattica a distanza, tutti i docenti italiani hanno dovuto ripensare al proprio modo di insegnare e di valutare: sono stati costretti a mettersi nuovamente in gioco e a sviluppare competenze per attivare modalità coinvolgenti ed interattive in una situazione contingente di profonda e improcrastinabile trasformazione.

La $\mathrm{DaD}$, infatti, ha trasformato la didattica da trasmissiva ad attiva promuovendo pratiche e metodologie, utilizzando strumenti, e prevedendo ambienti che fossero quanto più flessibili ed efficaci a raggiungere tutti gli studenti coinvolti. Il docente, per diventare un designer di un ambiente inclusivo nel rispetto delle tre dimensioni sopracitate, ha dovuto:

- preparare materiali didattici digitali adeguati;

- sviluppare percorsi individualizzati e personalizzati, volti a far raggiungere a tutti gli obiettivi comuni e a far sviluppare le peculiari potenzialità intellettive di ciascuno nel rispetto delle differenze;

- trovare compiti significativi che stimolassero la riflessione, la discussione attraverso feedback aperti e costruttivi e consentissero una valutazione formativa, ovvero una valutazione attenta ai processi ed ai percorsi evolutivi.

L'esperienza della $\mathrm{DaD}$ ha in molti casi (purtroppo non in tutti) permesso di capire che la didattica digitale non si può tradurre in un utilizzo delle nuove tecnologie viste come semplici strumenti, ma è necessario pensare ad essa come una dimensione integrante, una risorsa aggiuntiva utile a creare un ambiente di apprendimento maggiormente inclusivo e collaborativo. 


\section{Gli strumenti digitali}

Nell'ambiente di apprendimento digitale gli studenti possono scoprire una loro nuova dimensione di cittadinanza, quella, appunto, digitale. Imparano a rispettare diverse tipologie di regole e a salvaguardare i propri diritti in un contesto virtuale e sperimentano nuove modalità di apprendimento collaborativo attraverso l'uso di strumenti e piattaforme che permettono la costruzione collettiva di materiali. In particolare risultano efficaci per l'apprendimento cooperativo a distanza i seguenti strumenti:

\section{Canva}

È uno strumento creativo e stimolante che permette agli studenti di collaborare alla realizzazione di infografiche, volantini, brochure e molto altro.

\section{Google documenti}

Attraverso la condivisione di un medesimo documento, gli studenti possono collaborare attivamente alla stesura di un testo anche in modalità sincrona

\section{Tes blendspace}

È uno strumento applicativo che permette di organizzare immagini e pensieri in modalità cooperativa, raccogliendo materiali di varia natura (file di testo, immagini, video, mappe,...) su una bacheca virtuale.

\section{Il laboratorio della Summer}

Nel workshop della Summer School è stato proposto ai docenti di utilizzare gli strumenti sopra descritti. La finalità dell'azione, tuttavia, non voleva essere quella di spiegare l'utilizzo di uno strumento digitale, ma quella di creare un ambiente di apprendimento inclusivo utilizzando gli strumenti digitali. I docenti hanno dovuto far fronte ad un "compito" specifico, collaborando, mettendo a disposizione le proprie competenze, ragionando insieme per trovare la soluzione migliore per rispondere alla richiesta: come in un gioco di ruoli, si sono trovati nella posizione di "studenti" e hanno potuto apprezzare la forza e il valore dell'inclusione.

Per dare concretezza e valore al lavoro cooperativo il workshop prevedeva la creazione di tre gruppi, ciascuno dei quali ha ricevuto un compito da realizzare in un ambiente di apprendimento digitale in modalità collaborativa. Lo stimolo di partenza è stato per tutti i frequentanti la visione di un'immagine cui è seguita la somministrazione di un compito:

1. "Tutto in una valigia": prevedeva la realizzazione di una timeline delle migrazioni attraverso un'infografica, utilizzando la piattaforma CANVA.

2. "Se fossi...": chiedeva di potersi immedesimarsi nei panni di un"diverso" da sé e realizzare un'intervista utilizzando un documento di GOOGLE condiviso.

3. "Tutti per uno": prevedeva di esprimere le proprie emozioni attraverso immagini, video, file di testo utilizzando la piattaforma TESBLENDSPACE.

Dopo il lavoro condiviso, ogni gruppo ha avuto la possibilità di illustrare quanto prodotto, raccontare come si era svolto il lavoro e mettendo in luce eventuali difficoltà incontrate nell'utilizzo di una nuova applicazione digitale. Il confronto finale è stato utile, poi, per evidenziare 
le potenzialità dei nuovi ambienti di apprendimento sperimentati, e di comprendere se quella modalità collaborative a distanza fosse stata in grado di attivare dinamiche di inclusività.

Vengono messe a disposizione di tutti i lettori di Novecento.org sia la presentazione sul valore inclusivo dell'ambiente di apprendimento, che illustra come le tre dimensioni si orchestrino sia nella didattica in presenza sia nella didattica a distanza sia le attività proposte con le immagini ed $\mathrm{i}$ videotutorial dei tre strumenti.

Tali attività possono offrire una buona base di partenza per progettare nuovi percorsi in classe per l'insegnamento di Educazione Civica, in ambienti che non si limitino ad offrire esclusivamente strumenti compensativi e misure dispensative, ma che siano attenti a sviluppare i saperi, le abilità e le competenze di tutti gli studenti.

Note:

[1] Jürgen Habermas, L'inclusione dell'altro. Studi di teoria politica, tr. it a cura di L. Ceppa, Feltrinelli, Milano 1998

[2] Sancassani ha ben delineato il passaggio dal paradigma del Docente Oratore a quello del Docente Designer: «si tratta di passare dalla responsabilizzazione riguardo una corretta ed esaustiva esposizione dei contenuti alla responsabilizzazione sulla progettazione e gestione di un'esperienza che supporti nel miglior modo possibile il raggiungimento dei Risultati di Apprendimento Attesi». S. Sancassani con F. Brambilla, D. Casiraghi, P. Marenghi, Progettare l'innovazione didattica, Pearson, Milano-Torino, 2019. 